Received 00th January 20xx, Accepted 00th January 20xx DOI: $10.1039 / \times 0 \times x 00000 x$

\section{Thionation induces efficient generation of excited triplet state and singlet oxygen: heavy atom-free BODIPY photosensitizers based on $S_{1}\left(n, \pi^{*}\right)$ state}

\author{
Wenbin Hu, ${ }^{a}$ Xian-Fu Zhang ${ }^{* b}$ and Mingyu Liu ${ }^{a}$
}

The attachement of SR ( $R$ is alkyl or phenyl) makes BODIPY efficiently generate excited triplet state $\left(T_{1}\right)$ and singlet oxygen with quantum yield up to 0.82 (compared to 0.10 of parent BODIPY). This affords an efficient method to synthesize heavy atom free photosensitizers. This efficient $T_{1}$ formation is because SR presence leads to a new excited state $S_{1}\left(n, \pi^{*}\right)$ with an energy smaller but close to the usual $S_{1}\left(\pi, \pi^{*}\right)$ state.

The excited triplet states of organic materials have become the focus of several photoinitiated processes, such as photosensitized singlet oxygen generation for PDT (photodynamic therapy of tumor), ${ }^{1-3}$ photochemical upconversion of near IR sunlight, ${ }^{4-6}$ photoredox catalysis, ${ }^{7-8}$ and singlet fission for efficient electricity generation in solar cell. ${ }^{9}$ In many poly aromatic hydrocarbons, however, triplet excited states are often difficult to generate through direct photoexcitation owing to: (1) Intersystem crossing (ISC) from $S_{1}\left(\pi, \pi^{*}\right)$ to $T_{1}\left(\pi, \pi^{*}\right)$ is symmetrically forbidden, (2) the large singlettriplet energy splitting in their chromophores. ${ }^{10}$ Thus either triplet sensitization or chemical modification is used to populate triplet state. Triplet sensitization is through triplet energy transfer from a triplet donor to a target acceptor compound to efficiently access its triplet manifold of the acceptor. ${ }^{11}$ Chemical methods to enhance the triplet formation include:

(1) Incorporation of heavy atoms into a target organic compound, such as iodine or bromine atom substitution, second- or third-row transition metal ion complexion with a target organic chromophore. This is because the spin-orbit coupling (SOC) constant is directly proportional to atomic number $Z ;^{1,12}$ (2) Appending organic electron donors to the target molecule, since photo excitation can induce the formation of charge separation state, and then charge recombination generate excited triplet state. ${ }^{13}$

\footnotetext{
a. Department of Chemical Engineering, Hebei Normal University of Science and Technology, Qinhuangdao, Hebei Province, China, 066004

b. MPC Technologies, Hamilton, Ontario, Canada L8S $3 \mathrm{H} 4$.

Electronic Supplementary Information (ESI) available: [methods of NPs preparation, singlet oxygen detection and quantification]. See DOI: 10.1039/x0xx00000x
}
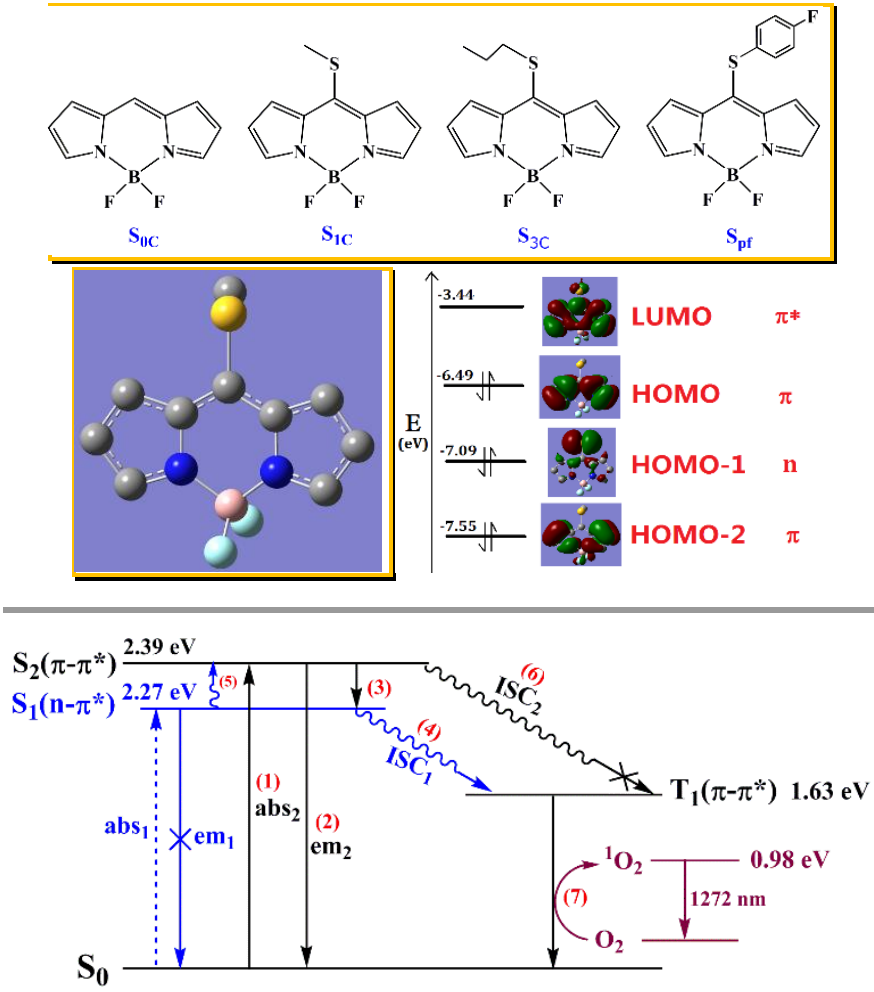

Fig. 1. Top: Chemical structures and abbreviations of SR substituted BODIPYs. Middle: The calculated geometry and frontier molecular orbitals for $\mathrm{S}_{1 \mathrm{c}}$. Bottom: Mechanism that makes SR-BODIPY efficiently generate the excited triplet state; abs: absorption, em: emission, ISC: intersystem crossing.

However, heavy-atom presence also drastically shortens triplet lifetimes, while donor-acceptor architectures can be very cumbersome and tedious to design and synthesize. Another important issue is that the second- and third-row transition metals are relatively low natural abundance and requires significant cost. Therefore new methods for efficient generation of excited triplet states are highly desirable and have been under extensive investigation. Parent BODIPY $\mathbf{S}_{0 \mathrm{c}}$ is very highly fluorescent but shows low ability in generating excited triplet 

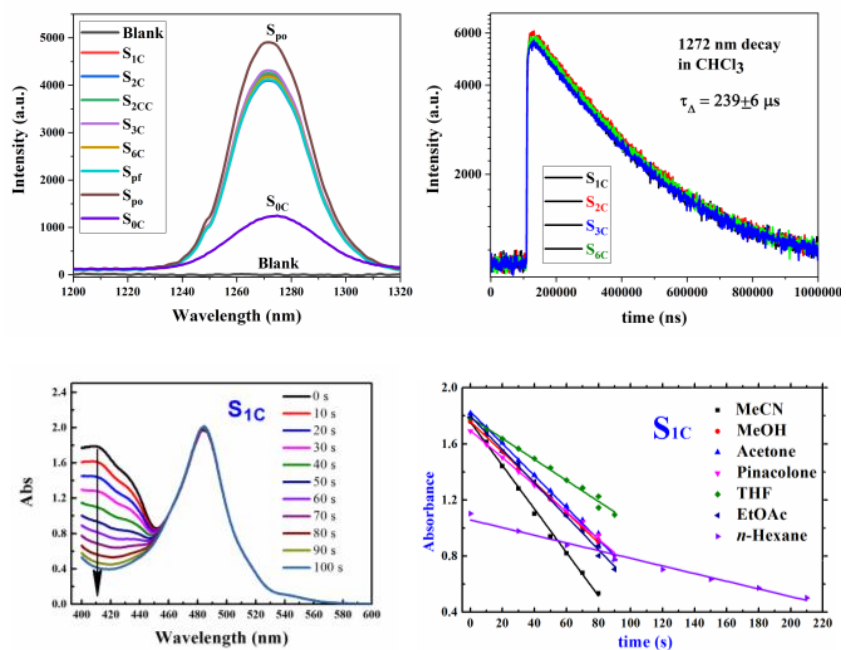

Fig. 2. Top: the NIR emission of singlet oxygen and its decay at $1272 \mathrm{~nm}$ in air saturated $\mathrm{CHCl}_{3}$ solution of SR-BODIPY with excitation at $490 \mathrm{~nm}$ (absorbance 0.50 ). Bottom Left: the decrease of the DPBF absorption in the presence of $\mathrm{S}_{1 \mathrm{C}}$ in MeCN with irradiation at $490 \mathrm{~nm}$ (70 W Xenon lamp with a monochromator). Bottom Right: The linea dependence of DPBF absorbance at $410 \mathrm{~nm}$ on time.

state. ${ }^{14}$ Nevertheless, modified BODIPYS have been proposed to act as singlet oxygen photosensitizers for PDT. ${ }^{15-19}$ We have also systematically and extensively studied how to make BODIPY generate excited triplet state $T_{1}$ efficiently by three methods: attaching an electron donor, ${ }^{20-26}$ synthesizing BODIPY dimer, ${ }^{27}$ ${ }^{29}$ and $\mathrm{I} / \mathrm{Br}$ substitution. ${ }^{30}$

In this report, we show that thioalkyl or thiophenyl substitution can also lead to efficient excited triplet state formation for BODIPY compounds (Fig. 1). The mechanism for triplet formation by this method is also revealed by various methods (Fig. 1 bottom).

Table 1. $\Phi_{\Delta}$ values in different solvents**

\begin{tabular}{|c|c|c|c|c|c|}
\hline$\varepsilon$ & solvent & $\mathbf{S}_{1 \mathrm{C}}$ & $\mathbf{S}_{3 \mathrm{C}}$ & $S_{p f}$ & $\mathbf{S}_{0 \mathrm{C}}$ \\
\hline 36.6 & $\mathrm{CH}_{3} \mathrm{CN}$ & 0.60 & 0.64 & 0.15 & 0.12 \\
\hline 33. 0 & $\mathrm{CH}_{3} \mathrm{OH}$ & 0.39 & 0.34 & 0.50 & 0.082 \\
\hline 20.7 & Acetone & 0.30 & 0.58 & 0.83 & 0.13 \\
\hline 12.8 & Pinacolone & 0.50 & 0.45 & 0.63 & NM \\
\hline 7.52 & THF & 0.40 & 0.25 & 0.70 & 0.071 \\
\hline 6.02 & EtOAc & 0.44 & 0.27 & 0.51 & 0.13 \\
\hline 4.81 & Chloroform & 0.41 & 0.42 & 0.40 & 0.13 \\
\hline 2.02 & n-hexane & 0.090 & 0.17 & 0.54 & 0.12 \\
\hline
\end{tabular}

** $\varepsilon$ : dielectrical constant of a solvent. NM=not measured.

Parent BODIPY is known to exhibit low capability to generate singlet oxygen (Table 1). In contrast, all SR-BODIPYs show much higher ability as shown in Fig. 2, in which the intensity of 1270 $\mathrm{nm}$ band (NIR phosphorescence of ${ }^{1} \mathrm{O}_{2}$ ) is ranked by $\mathrm{S}_{0 \mathrm{C}}<<\mathrm{S}_{1 \mathrm{C}} \sim$ $\mathrm{S}_{3 \mathrm{C}}<\mathrm{S}_{\mathrm{pf}}$ (these spectra have been recorded under the same conditions: absorbance of an SR-BODIPY is 0.50 at excitation wavelength $490 \mathrm{~nm}$ in $\mathrm{CHCl}_{3}$ ), leading to the much higher quantum yield of singlet oxygen formation ( $\Phi_{\Delta}$ in Table 1$)$. The $1270 \mathrm{~nm}$ emission of the SR-BODIPYs is indeed due to $\mathrm{O}_{2}\left({ }^{1} \Delta_{\mathrm{g}}\right)$, since both the peak position $1270 \mathrm{~nm}$ and band shape, as well as the emission lifetime $(239 \pm 6 \mu \mathrm{s})$ are all well consistent with that of reported for $\mathrm{O}_{2}\left({ }^{1} \Delta_{\mathrm{g}}\right) \cdot{ }^{31}$ On the other hand, if either
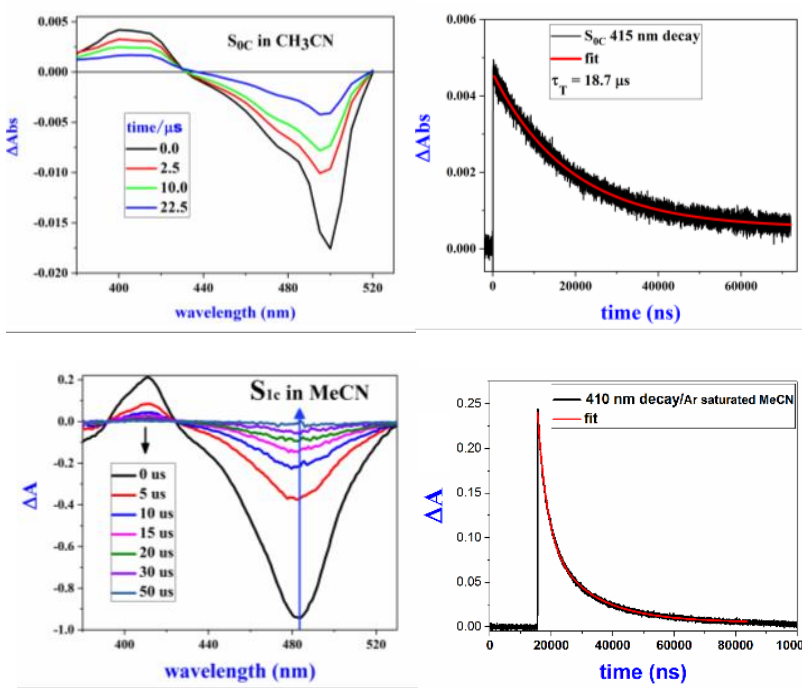

Fig. 3. Left Column: The time resolved $T_{1}-T_{n}$ transient absorption spectra of compound $\mathrm{S}_{0 \mathrm{C}}$ and $\mathrm{S}_{1 \mathrm{C}}\left(10 \mu \mathrm{M}\right.$ in $\left.\mathrm{CH}_{3} \mathrm{CN}\right)$; Right Column: TA decay at $410 \mathrm{~nm}$ in Ar-saturated $\mathrm{CH}_{3} \mathrm{CN}$. All the solutions are excited with OPO laser at $485 \mathrm{~nm}$ ( $4 \mathrm{~ns}$ and $\sim 5 \mathrm{~mJ}$ per pulse).

oxygen or SR-BODIPY is absent, the $1270 \mathrm{~nm}$ NIR band disappears. These results imply that SR-BODIPYs indeed act as the photosensitizers of singlet oxygen.

The photosensitizing ability of the SR-BODIPYs are further demonstrated by chemical trapping, using DPBF (diphenylisobenzofuran) as the trapper of singlet oxygen. The UV-Vis absorption of DPBF ( $410 \mathrm{~nm}$ band) decreases in the co-presence of a SR-BODIPY, air and $490 \mathrm{~nm}$ light irradiation, during which the absorption of the SR-BODIPYs shows no change (Fig. 2 Bottom). Control experiments show that the photodecomposition of DPBF does not occur if any of SRBODIPY, oxygen and $490 \mathrm{~nm}$ light irradiation is absent. The plot of absorbance at $410 \mathrm{~nm}$ against time is linear from which the reaction rate constant could be obtained to calculate $\Phi_{\Delta}$.

These SR-BODIPYs exhibit significantly high $\Phi_{\Delta}$ up to 0.83 in various solvents (Table 1 ). For example, $\Phi_{\Delta}$ of $S_{p f}$ is $0.83,0.70$ and 0.63 in acetone, THF and pinacolone respectively; while $\Phi_{\Delta}$ of $S_{3 c}$ is $0.64,0.58$ and 0.45 in acetonitrile, acetone and pinacolone respectively. All of which are all much higher than that of $S_{0 c}$ ( 0.11 in average in different solvents), clearly indicating that SR presence effectively makes BODIPYs become good singlet oxygen photosensitizer.

Next we examined if SR enhances the triplet excited state formation. Fig. 3 gives the transient absorption spectra (TAS) in acetonitrile (argon saturated) with OPO laser (4 ns pulsed) excitation at $485 \mathrm{~nm}$. The TAS of $\mathrm{S}_{0 c}$ is the same as that reported previously, ${ }^{32}$ it is known that the positive band peaked at 410 $\mathrm{nm}$ is due to triplet-triplet $\left(T_{1}-T_{n}\right)$ transient absorption, and the negative band is due to the bleaching of the ground state $S_{0}$. For $S_{1 c}$, the shape of TAS is very similar to that of $S_{0 c}$, except the slight red shift in peak positions. The negative absorption is assigned to the bleaching of the ground state $S_{0}$, since their band shape and position matches that of ground state $S_{0}$. The positive spectra (maxima $412 \mathrm{~nm}$ ) are assigned to $T_{1}$ to $T_{n}$ absorption, and the decrease of the positive spectra reflects the 
decay of $T_{1}$ state with time $\left(T_{1} \rightarrow S_{0}\right)$. The rise of the negative band occurs concomitantly with the decrease of positive band, this is due to the regeneration of $\mathrm{S}_{0}$ from $\mathrm{T}_{1}$ decay $\left(\mathrm{T}_{1} \rightarrow \mathrm{S}_{0}\right)$, this $\mathrm{T}_{1} \rightarrow \mathrm{S}_{0}$ conversion process is also supported by the presence of an isobestic point at $425 \mathrm{~nm}$.

In comparison to $\mathrm{S}_{0 \mathrm{c}}, \mathrm{S}_{1 \mathrm{c}}$ shows much larger absorption intensity (positive peak $\Delta \mathrm{A}$ value in Fig. 3 ) in TAS under the same condition, suggesting that $S_{1 c}$ generates excited triplet state $T_{1}$ much more efficiently, i.e. SR presence very significantly enhances the excited triplet state formation. This result is consistent with the singlet oxygen measurement results. Since singlet oxygen is generated by $\mathrm{T}_{1}$ via energy transfer: $\mathrm{T}_{1}+\mathrm{O}_{2} \rightarrow$ $\mathrm{S}_{0}+{ }^{1} \mathrm{O}_{2}$, the more $\mathrm{T}_{1}$ is generated, the more singlet oxygen is formed.

The triplet lifetime $\left(\tau_{T}\right)$ of $S_{1 C}$ and $S_{3 C}$ is obtained from the $T_{1}$ decay curve at $410 \mathrm{~nm}$ in argon saturated solvent (Fig. 3 right column). $\tau_{\mathrm{T}}$ of $\mathrm{S}_{1 \mathrm{C}}$ is $15.5 \mu \mathrm{s}$, which is close to $18.7 \mu \mathrm{s}$ of $\mathrm{S}_{\mathrm{OC}}$. Molecular oxygen quenches $\mathrm{T}_{1}$ very significantly $\left(\mathrm{T}_{1}+\mathrm{O}_{2} \rightarrow \mathrm{S}_{0}+\right.$ ${ }^{1} \mathrm{O}_{2}$ ), since $\mathrm{T}_{1}$ decays much faster in the presence of oxygen (supporting info), $\tau_{\mathrm{T}}$ of $\mathrm{S}_{1 \mathrm{C}}$ and $\mathrm{S}_{\mathrm{OC}}$ in air saturated solvent is 0.24 and $0.23 \mu \mathrm{s}$, respectively, this oxygen quenching provides a further evidence that the positive signal is due to $T_{1}$. The rate constant of the oxygen quenching $\left(\mathrm{k}_{\mathrm{q}}\right)$ can be computed by: $\tau_{\mathrm{T}}{ }^{0} / \tau_{\mathrm{T}}=1+\mathrm{k}_{\mathrm{q}} \tau_{\mathrm{T}}{ }^{0}\left[\mathrm{O}_{2}\right]$. The obtained $\mathrm{k}_{\mathrm{q}}$ value for $\mathrm{S}_{1 \mathrm{c}}$ and $\mathrm{S}_{\mathrm{OC}}$ is $2.05 \times 10^{9}$ and $2.15 \times 10^{9} \mathrm{M}^{-1} \mathrm{~s}^{-1}$, respectively. The large $\mathrm{k}_{\mathrm{q}}$ values also supports that the positive signal is due to $T_{1}$.

To understand why SR presence effectively enhances $T_{1}$ and singlet oxygen formation, we have studied the related photophysical processes and calculated their electronic properties. In Fig. 4, we compare the absorption spectra of SRBODIPYs with that of unsubstituted $\mathrm{S}_{\text {oc. }}$ A new absorption shoulder appears at $\sim 513 \mathrm{~nm}$ for each SR-BODIPY in all solvents studied, in addition to the main peak at $\sim 89 \mathrm{~nm}$. The new peak features a weak absorption and a $\sim 15 \mathrm{~nm}$ red shift relative to the main peak.

To reveal the nature of this new peak, we have performed quantum chemical calculations using time dependent density functional theory. Table 2 shows the results, together with the optimized geometry and molecular orbitals in Fig. 1. For HOMO, LUMO and HOMO-2, each orbital is delocalized over two pyrrole rings (Fig. 1), therefore they are $\pi$ orbitals. For HOMO-1, however, it is almost localized on the $\mathrm{S}$ atom, it is therefore an $\mathrm{n}$ orbital. The calculated bands in Table 2 are consistent with the measured UV-Vis absorption (Fig. 4). In particular, the $512 \mathrm{~nm}$ band is assigned to $\mathrm{n} \rightarrow \pi *$ transitions (97\%) with a small oscillation strength of 0.018 , while the main band at $485 \mathrm{~nm}$ is $\pi \rightarrow \pi *$ transitions (98\%) with a large oscillation strength of 0.43 .

Fig. 4 also compares the fluorescence emission of SRBODIPYs with that of Soc. The spectral shape of emission is similar to that of $S_{0 C}$ but the peak position is red shifted $25 \mathrm{~nm}$. No new emission peaks occur, suggesting $S_{1}\left(n, \pi^{*}\right)$ is non emissive because $S_{1}\left(n, \pi^{*}\right) \rightarrow S_{0}\left(\pi, \pi^{*}\right)$ emission is symmetry forbidden. The excitation spectrum is the same as that of absorption spectrum. The fluorescence quantum yield $\left(\Phi_{\mathrm{f}}\right)$, however, shows a large decrease, for example, in chloroform $\Phi_{f}$ of $S_{0 c}$ is 0.95 , but $\Phi_{f}$ of $S_{1 c}, S_{3 c}$, and $S_{p f}$ is
Table 1. The calculated first five UV-Vis absorption bands for $\mathrm{S}_{1 \mathrm{C}}$ in $\mathrm{CH}_{3} \mathrm{CN}^{* * *}$

\begin{tabular}{|c|c|c|c|c|}
\hline Absorption Band $\mathrm{i}$ & Transition Assignment & orbitals & $\lambda_{\text {abs }}(\mathrm{nm})$ & $f$ \\
\hline \multirow[t]{2}{*}{ Excited State 1} & HOMO-1 $\rightarrow$ LUMO (97\%) & $\mathrm{n} \rightarrow \pi^{*}$ & 512 & 0.018 \\
\hline & HOMO $\rightarrow$ LUMO (3\%) & $\pi \rightarrow \pi *$ & & \\
\hline \multirow[t]{3}{*}{ Excited State 2} & HOMO-2 $\rightarrow$ LUMO $(10 \%)$ & $\pi \rightarrow \pi^{*}$ & 485 & 0.43 \\
\hline & HOMO $\rightarrow$ LUMO (88\%) & $\pi \rightarrow \pi *$ & & \\
\hline & HOMO-1 $\rightarrow$ LUMO (2\%) & $\mathrm{n} \rightarrow \pi *$ & & \\
\hline \multirow[t]{2}{*}{ Excited State 3} & HOMO-1 $\rightarrow$ LUMO (90\%) & $\mathrm{n} \rightarrow \pi^{*}$ & 402 & 0.21 \\
\hline & HOMO $\rightarrow$ LUMO (10\%) & $\pi \rightarrow \pi *$ & & \\
\hline Excited State 4 & HOMO-2 $\rightarrow$ LUMO (99\%) & $\pi \rightarrow \pi *$ & 385 & 0.039 \\
\hline \multirow[t]{2}{*}{ Excited State 5} & HOMO-4 $\rightarrow$ LUMO (6\%) & $\pi \rightarrow \pi *$ & 297 & 0.18 \\
\hline & HOMO-3 $\rightarrow$ LUMO (91\%) & $\pi \rightarrow \pi *$ & & \\
\hline
\end{tabular}

$* * *: \lambda_{\text {abs }}$ is the absorption maximum*1.14. $\mathrm{f}$ is the oscillation strength.

$0.52 \pm 0.05 \mathrm{~ns}$, which is about a $45 \%$ decrease. This large decrease indicates that the presence of SR causes the increase of either intersystem crossing rate constant $\mathrm{k}_{\text {isc }}$ or $\mathrm{k}_{\text {ic }}$ (internal conversion rate constant), according to following equations:

$\Phi_{\mathrm{f}}=\mathrm{k}_{\mathrm{f}} /\left(\mathrm{k}_{\mathrm{f}}+\mathrm{k}_{\text {isc }}+\mathrm{k}_{\text {ic }}\right)=\mathrm{k}_{\mathrm{f}} \tau_{\mathrm{f}}$,

$\tau_{f}=1 /\left(k_{f}+k_{\text {isc }}+k_{\text {ic }}\right)$,

in which $k_{f}$ and $\tau_{f}$ is the rate constant of radiation, and fluorescence lifetime, respectively.

SR substitution also leads to faster fluorescence decay of SRBODIPYs than that of $\mathrm{S}_{0 \mathrm{c}}$, as shown in Fig. 4 bottom, therefore SR-BODIPYs exhibit shorter fluorescence lifetime. For example, in $\mathrm{CHCl}_{3} \tau_{f}$ of $\mathrm{S}_{0 \mathrm{c}}$ is $7.33 \mathrm{~ns}$, while $\tau_{\mathrm{f}}$ of $\mathrm{S}_{1 \mathrm{C}}, \mathrm{S}_{3 \mathrm{C}}$, and $\mathrm{S}_{\mathrm{pf}}$ is $5.14 \pm 0.40$ $\mathrm{ns}$, which is a $30 \%$ decrease. On the other hand, $\mathrm{k}_{\mathrm{f}}$ of $\mathrm{S}_{1 \mathrm{c}}, \mathrm{S}_{3 \mathrm{c}}$, and $S_{p f}$ (calculated by $k_{f}=\Phi_{f} / \tau_{f}$ ) is $0.11 \times 10^{9} \mathrm{~s}^{-1}$, which is only slightly smaller than $k_{f}$ of $S_{1 c}\left(0.13 \times 10^{9} \mathrm{~s}^{-1}\right)$.

Based on above facts, we propose the mechanism, as shown in Fig. 1 Bottom, in which the blue part drives efficient $T_{1}$ formation ( $\mathrm{ISC}_{1}$ is efficient), while the black part is the regular
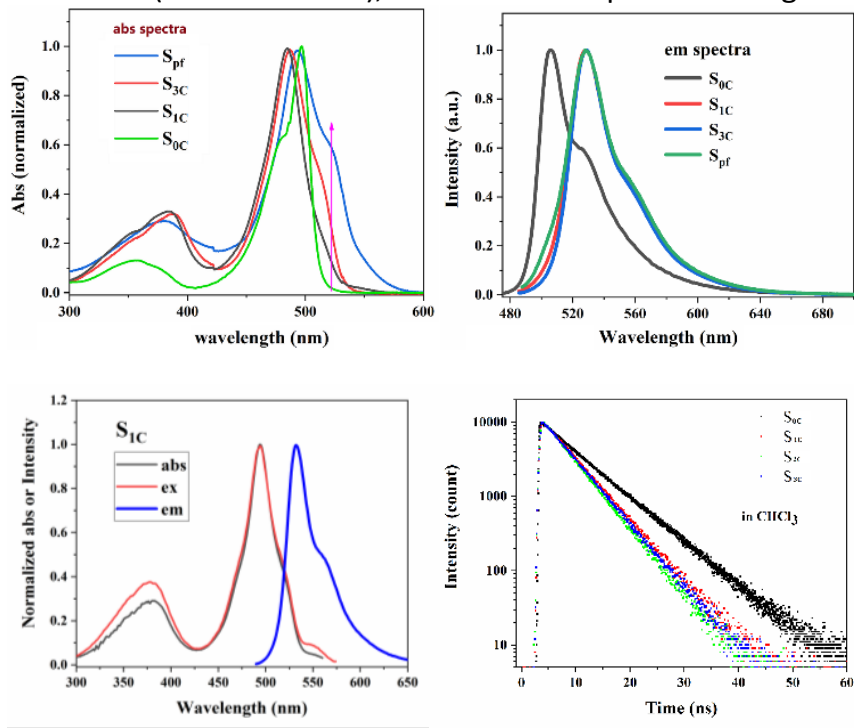

Fig. 4. Top: The normalized absorption and emission spectra of SR-BODIPYs. Bottom left: The normalized absorption, excitation and emission spectra of $\mathrm{S}_{1 \mathrm{C}}$; Bottom right: fluorescence decay at $540 \mathrm{~nm}$. Solvent: $\mathrm{CHCl}_{3}$, sample concentration $\sim 10 \mu \mathrm{M}$, excitation wavelength: $470 \mathrm{~nm}$ for em spectra, emission wavelength for ex spectra: $575 \mathrm{~nm}$. 
photophysical processes (ISC 2 is not efficient). When a meso-SR is attached onto the core of BODIPY, a new excited state $S_{1}\left(n, \pi^{*}\right)$ occurs, its energy is smaller but close to the usual $S_{2}\left(\pi, \pi^{*}\right)$ state (corresponding to $\mathrm{S}_{1}\left(\pi, \pi^{*}\right)$ in parent BODIPY). Due to the forbidden symmetry, absorption abs from $S_{0}\left(\pi, \pi^{*}\right)$ to $S_{1}\left(n, \pi^{*}\right)$ is fairly weak, and the emission em from $S_{1}\left(n, \pi^{*}\right)$ to $S_{0}\left(\pi, \pi^{*}\right)$ does not occur. On the contrary, absorption abs 2 from $S_{0}\left(\pi, \pi^{*}\right)$ to $S_{2}\left(\pi, \pi^{*}\right)$ is strong, and the emission em from $S_{2}\left(\pi, \pi^{*}\right)$ to $\mathrm{S}_{0}\left(\pi, \pi^{*}\right)$ is symmetry allowed. $S_{1}\left(n, \pi^{*}\right)$ is mainly formed from $S_{2}\left(\pi, \pi^{*}\right)$, because $S_{2}\left(\pi, \pi^{*}\right)$ tends to relax to lower $S_{1}\left(n, \pi^{*}\right)$ to stabilize the excited molecular structure (process 3 ). Intersystem crossing (ISC) from $S_{1}\left(n, \pi^{*}\right)$ to $T_{1}\left(\pi, \pi^{*}\right)$ is symmetrically allowed, but ISC from $S_{2}\left(\pi, \pi^{*}\right)$ to $T_{1}\left(\pi, \pi^{*}\right)$ is symmetrically forbidden, this explains why $S R$ enhances $T_{1}$ formation and renders BODIPY efficient photosensitizers for generating singlet oxygen. Fig. 4 shows that the intensity of $\mathrm{S}_{1}\left(\mathrm{n}, \pi^{*}\right)$ absorption for $\mathrm{S}_{\mathrm{pf}}$ is larger than that of $\mathrm{S}_{3 \mathrm{C}}$ and $\mathrm{S}_{1 \mathrm{C}}$, this explains why $S_{p f}$ is more efficient in singlet oxygen formation.

We have shown that thioalkyl and thiophenyl substitution makes BODIPY become efficient photosensitizer for excited triplet state and singlet oxygen formation. This is because a new $\mathrm{S}_{1}\left(\mathrm{n}, \pi^{*}\right)$ state appears and has a slightly lower energy than the usual $S_{1}\left(\pi, \pi^{*}\right)$ state in $S_{0 c}$ when SR is introduced onto BODIPY core. Since Intersystem crossing (ISC) from $S_{1}\left(n, \pi^{*}\right)$ to $T_{1}\left(\pi, \pi^{*}\right)$ is symmetrically allowed, while ISC from $S_{2}\left(\pi, \pi^{*}\right)$ to $T_{1}\left(\pi, \pi^{*}\right)$ is symmetrically forbidden, this explains why SR renders BODIPY efficient photosensitizers. This fact provides a new method to efficiently generate excited triplet state and singlet oxygen without the involvement of heavy atoms for some compounds, especially BODIPY in this study. We believe this method will be helpful in developing new heavy atom free photosensitizers for photodynamic therapy, photochemical upconversion of near IR sunlight in solar cell, and photoredox catalysis.

\section{Conflicts of interest}

There are no conflicts to declare.

\section{Notes and references}

1. J. Zhao, W. Wu, J. Sun and S. Guo, Chem. Soc. Rev., 2013, 42 , 5323-5351.

2. J. Zhao, K. Xu, W. Yang, Z. Wang and F. Zhong, Chem. Soc Rev., 2015, 44, 8904-8939.

3. M. Lan, S. Zhao, W. Liu, C.-S. Lee, W. Zhang and P. Wang Adv. Healthcare Mater., 2019, 8, 1900132.

4. T. F. Schulze and T. W. Schmidt, Energy Environ. Sci., 2015, 8, 103-125.

5. T. N. Singh-Rachford and F. N. Castellano, Coord. Chem. Rev. 2010, 254, 2560-2573.

6. Y. Y. Cheng, B. Fückel, R. W. MacQueen, T. Khoury, R. G. C. R. Clady, T. F. Schulze, N. J. Ekins-Daukes, M. J. Crossley, B. L. Stannowski, K. and T. W. Schmidt, Energy Environ. Sci. 2012, 5, 6953-6959.

7. B. D. Ravetz, A. B. Pun, E. M. Churchill, D. N. Congreve, T. Rovis and L. M. Campos, Nature, 2019, 565, 343-346.

8. A. K. Pal, C. Li, G. S. Hanan and E. Zysman-Colman, Angew. Chem., Int. Ed., 2018, 57, 8027-8031.
9.

10. M. Montalti, A. Credi, L. Prodi and M. T. Gandolfi, Handbook

J. Xia, S. N. Sanders, W. Cheng, J. Z. Low, J. Liu, L. M. Campos and T. Sun, Adv. Mater., 2017, 29, 1601652. of photochemistry. 3rd ed.;, CRC/Taylor \& Francis, Boca Raton, FL, 2006.

11. T. F. Schulze and T. W. Schmidt, Energy Environ. Sci., 2015, 8, 103-125.

12. J. Zhao, S. Ji, W. Wu, W. Wu, H. Guo, J. Sun, H. Sun, Y. Liu, Q Li and L. Huang, RSC Adv. , 2012, 2, 1712-1728.

13. J. Zhao, K. Chen, Y. Hou, Y. Che, L. Liu and D. Jia, Org. Biomol. Chem., 2018, 16, 3692-3701.

14. X.-F. Zhang and J. Zhu, J. Luminesc., 2019, 205, 148-157.

15. Q. Guan, L.-L. Zhou, Y.-A. Li and Y.-B. Dong, Chem. Commun., 2019, 55, 14898-14901.

16. Y. Hou, Q. Liu and J. Zhao, Chem. Commun., 2020, 56, 1721-1724.

17. Y. Liu, C. Xu, L. Teng, H.-W. Liu, T.-B. Ren, S. Xu, X. Lou, H. Guo, L. Yua and X.-B. Zhang, Chem. Commun., 2020, 56, 1956-1959.

18. W. Wang, L. Wang, Z. Li and Z. Xie, Chem. Commun., 2016, 52, 5402-5405.

19. Y. Zhou, R. C. H. Wong, G. Dai and D. K. P. Ng, Chem. Commun., 2020, 56, 1078-1081.

20. W. Hu, M. Liu, X.-F. Zhang, Y. Wang, Y. Wang, H. Lan and H. Zhao, J. Phys. Chem. C, 2019, 123, 15944-15955.

21. X.-F. Zhang and N. Feng, Chem. Asian J., 2017, 12, 24472456.

22. W. Hu, Y. Lin, X.-F. Zhang, M. Feng, S. Zhao and J. Zhang, Dyes Pigm., 2019, 164, 139-147.

23. X.-F. Zhang and N. Feng, Spectrochimica Acta Part A: Molecular and Biomolecular Spectroscopy, 2018, 189, 1321.

$24 . \quad$ W. Hu, X.-F. Zhang, X. Lu, S. Lan, D. Tian, T. Li, L. Wang, S. Zhao, M. Feng and J. Zhang, J. Luminesc., 2018, 194, 185192.

25. W. Hu, X.-F. Zhang, X. Lu, S. Lan, D. Tian, T. Li, L. Wang, S. Zhao, M. Feng and J. Zhang, Dyes Pigm., 2018, 149, 306314.

26. X.-F. Zhang, Y. Zhang and B. Xu, J. Photochem. Photobiol. A: Chemistry, 2017, 349C, 197-206.

27. X.-F. Zhang, X. Yang and B. Xu, Phys. Chem. Chem. Phys., 2017, 19, 24792-24804.

28. X.-F. Zhang, Dyes Pigm., 2017, 146, 491-501.

29. W. Pang, X.-F. Zhang, J. Zhou, C. Yu, E. Hao and L. Jiao, Chem. Commun., 2012, 48, 5437-5439

30. X.-F. Zhang, J. Photochem. Photobiol. A: Chemistry, 2018, 355, 431-443.

31. E. Boix-Garriga, B. Rodríguez-Amigo, O. Planas and S Nonell, in Singlet Oxygen Applications in Biosciences and Nanosciences eds. S. Nonell and C. Flors, Royal Society of Chemistry, Cambridge, 2016, p. 31. X.-F. Zhang and J. Zhu, J. Lumin., 2019, 212, 286-292. 